\title{
The use of ratios and percentage changes in sports medicine: time for a rethink?
}

\section{Greg Atkinson and Alan M. Batterham}

\section{Health and Social Care Institute, School of Health and Social Care, Teesside University, UK}

Ratios and percentage change statistics are ubiquitous in scientific research. In the context of sports medicine, some examples are cycling efficiency indices (power output/energy expenditure), the hamstring/quadriceps ratio $(\mathrm{H}: \mathrm{Q})$ and the percentage change in artery diameter during a test of flow-mediated dilation (FMD\%). There are of course many other examples in psychology, physiology and biomechanics. One can see how the simplicity of ratios makes them so popular amongst researchers for 'normalising' measurements of one variable (e.g. hamstring strength) with respect to another variable (quadriceps strength), in the case of the $\mathrm{H}: \mathrm{Q}$ ratio. Nevertheless, ratios are not the only approach available to scale or normalise measurements, and unfortunately they can be very misleading in certain circumstances (1-3).

One important point is that percentage change statistics like FMD\% are, in fact, ratios since a change in any outcome (from baseline to follow-up), which is then expressed as a percentage of the initial value of that outcome, is synonymous with dividing the follow-up value by the baseline value. For example, a change in arterial diameter from $4.0 \mathrm{~mm}$ at baseline to $4.5 \mathrm{~mm}$ at post-ischemic peak gives a FMD\% of $12.5 \%$, which is synonymous with a follow-up/baseline ratio of 1.125. In this example, the follow-up measurement is the numerator of the ratio, and the baseline measurement is the denominator. 
We believe that the majority of researchers interested in sports medicine might not have appreciated that percentage change statistics are ratios and are, therefore, reliant on all the statistical assumptions that underlie ratios (3). Moreover, we doubt whether many researchers check that the ratios they are working with are actually appropriate for their data. Essentially, a ratio is based on the assumption that the slope of the relationship between logarithmically-transformed numerator and denominator is 1 (Figure 1). If this is not so, then the ratio will scale inaccurately at the lower and higher ends of the range of measured values, leading to errors in interpreting measurements on individuals and in samples (Figure 2). There are other problems. When one normally distributed variable is divided by another normally distributed variable, it is unlikely that the resulting ratio is normally distributed itself (1-3), thus making any parametric analyses trickier to handle.

So what is the best solution? It should be said that not all ratios might be inappropriate for scaling data, and one could explore underlying assumptions for the ratio one is working with. However, it is preferable in our opinion to have an analysis approach which is accurate in most circumstances, at least more circumstances than that of a ratio. In the context of randomised controlled trials, Vickers (5) has already shown that analysis of covariance is generally superior to a percentage change statistic. Controversially, Packard and Boardman (3) called for a complete end to the use of ratios and percent changes in an attempt to normalize physiological data for variation in body size. These authors also advised readers of scientific research not to place great confidence in results of studies that use ratios for scaling, but this advice has not been heeded properly. Like many other statisticians, Packard and Boardman (3) presented a good case for an analysis of covariance (ANCOVA) approach being preferable. 
An ANCOVA-based approach essentially involves entering the baseline value (or denominator of a ratio) as a covariate in the statistical model one is using to quantify effect sizes. This statistical model would include all the factors of interest in the study, e.g. sex if the HQR is to be compared between males and females. The numerator of the ratio or the difference between follow-up and baseline measurements could be the dependent variable in the model, although it is probably appropriate to logarithmically transform the data first. If logarithmic transformation of the data is found to be appropriate, the resulting estimates of mean change in outcome can be back-transformed into percentages for conventional interpretation. Important assumptions, such as 'parallelism' in ANCOVA could be checked, although Senn (4) has argued that ANCOVA is superior to simple change summary statistics like percentage changes and ratios in most cases.

We believe that an ANCOVA approach would likely result in more accurate estimates of ratios and percentage changes, especially when the relationship between baseline (denominator) and follow-up (numerator) does not equal 1. Statistical software such as SPSS support these types of analyses and we believe that the Generalized Linear models (for group comparisons) and Generalized Estimating Equations (for repeated measures data) options are most amenable to this sort of analysis. We encourage all submitting authors of IJSM to consider using these ANCOVA approaches if they are analysing percentage changes or ratios. Such good practice would be yet another unique aspect of the scientific rigour of IJSM.

\section{References}

1. Allison DB, Paultre F, Goran MI, PoehIman ET, Heymsfield SB. Statistical considerations regarding the use of ratios to adjust data. Int J Obes 19: 644-652, 1995. 
2. Atkinson G, Batterham AM, Black MA, Cable NT, Hopkins ND, Dawson EA, Thijssen $\mathrm{DH}$, Jones $\mathrm{H}$, Tinken $\mathrm{TM}$, and Green DJ. Is the ratio of flow-mediated dilation and shear rate a statistically sound approach to normalization in cross-sectional studies on endothelial function? J Appl Physiol 107: 1893-1899, 2009.

3. Packard GC, Boardman TJ (1999). The use of percentages and size-specific indices to normalize physiological data for variation in body size: wasted time, wasted effort? Comp Biochem Physiol Part A 122: 37-44

4. Senn, S. Change from baseline and analysis of covariance revisited. Statist. Med. 2006; 25:4334-4344

5. Vickers, A.J. (2001). The use of percentage change from baseline as an outcome in a controlled trial is statistically inefficient: a simulation study BMC Med Res Methodol $1: 6$

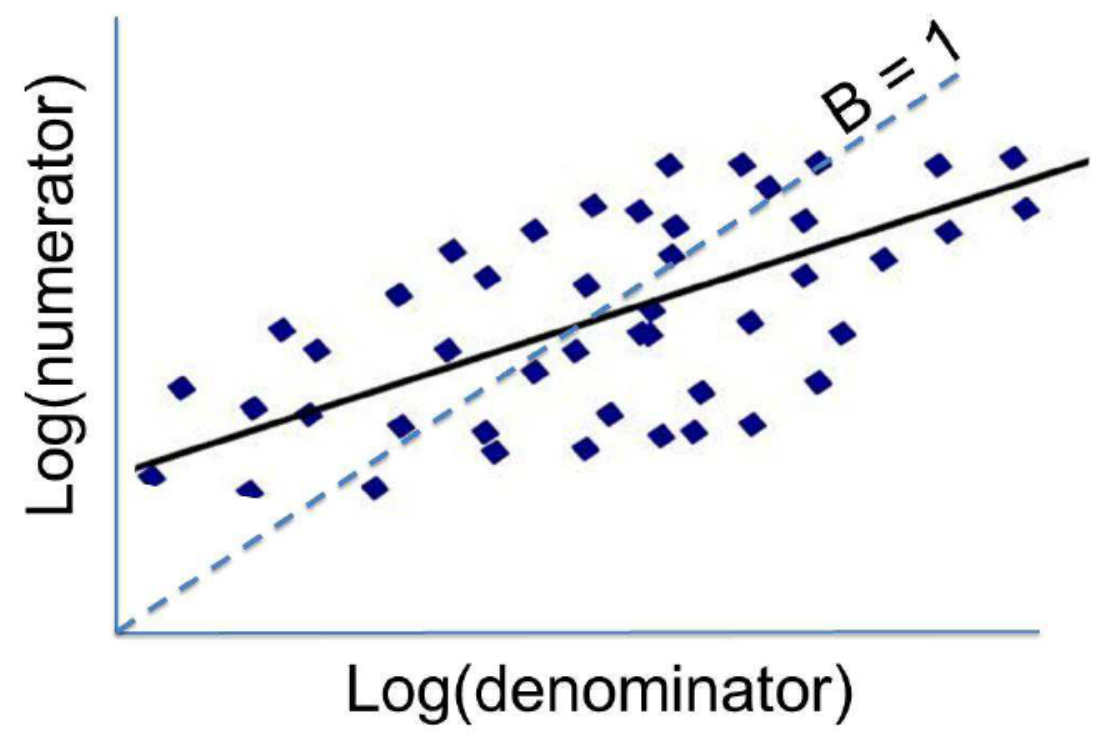

Figure 1. An example where a ratio might not be scaling consistently across the range of measured values of the denominator. If the slope (B) of the relationship between $\log$ (numerator) and $\log$ (denominator) deviates substantially from 1 (shown by the dashed blue line), then a simple ratio of numerator/denominator will likely not scale accurately across the range of measurements, especially towards each end of the range. In this case, an ANCOVA approach to the scaling or normalisation is preferable, where the logarithmically transformed denominator values are entered into a statistical model as a covariate and the logarithmically transformed numerator is the outcome. 


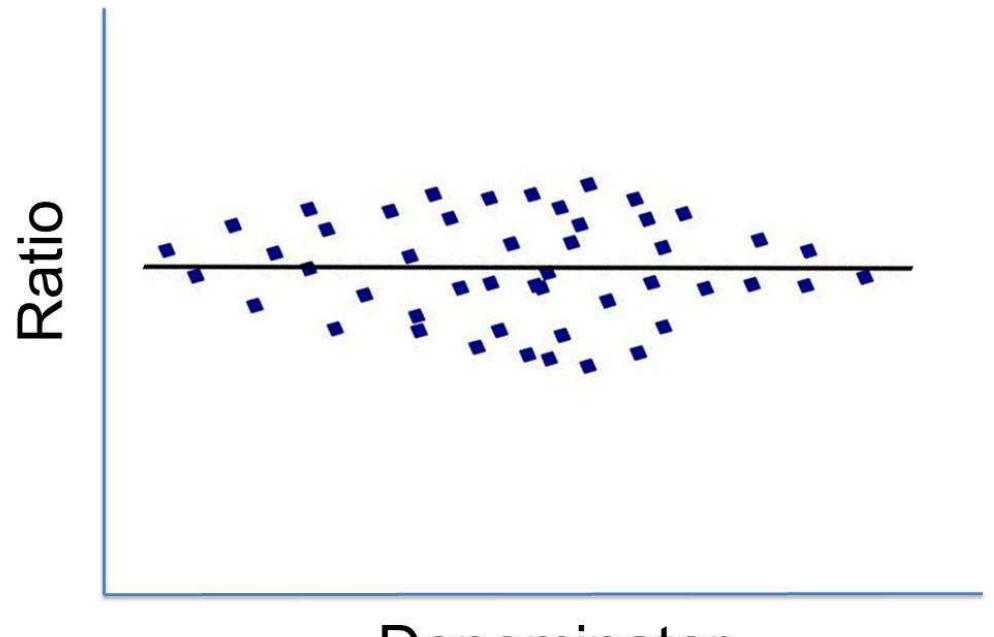

Denominator

Figure 2. This is the ideal situation in which a ratio is scaling consistently across the range of values of the denominator. Often the slope of this plot is not close to zero, indicating that the ratio would provide biased estimates at the lower and higher ends of the range. 